\title{
Tailoring Laser Propulsion for Future Applications in Space
}

\author{
Hans-Albert Eckel and Stefan Scharring \\ German Aerospace Center (DLR) - Institute of Technical Physics, Pfaffenwaldring 38 - 40, \\ 70569 Stuttgart, Germany
}

\begin{abstract}
Pulsed laser propulsion may turn out as a low cost alternative for the transportation of small payloads in future. In recent years DLR investigated this technology with the goal of cheaply launching small satellites into low earth orbit (LEO) with payload masses on the order of 5 to $10 \mathrm{~kg}$. Since the required high power pulsed laser sources are yet not at the horizon, DLR focused on new applications based on available laser technology. Space-borne, i.e. in weightlessness, there exist a wide range of missions requiring small thrusters that can be propelled by laser power. This covers space logistic and sample return missions as well as position keeping and attitude control of satellites.

First, a report on the proof of concept of a remote controlled laser rocket with a thrust vector steering device integrated in a parabolic nozzle will be given. Second, the road from the previous ground-based flight experiments in earth's gravity using a 100-J class laser to flight experiments with a parabolic thruster in an artificial 2D-zero gravity on an air cushion table employing a 1-J class laser and, with even less energy, new investigations in the field of laser micro propulsion will be reviewed.
\end{abstract}

Keywords: Laser propulsion, Free flight experiments, Steering, Lightcraft, Parabolic thruster, Impulse coupling coefficient, Remote control, Laser ablation, Microgravity, Air cushion table. PACS: 41.75.Jv, 42.15.Dp, 42.62.-b, 52.38.Mf.

\section{INTRODUCTION}

Since the invention of laser propulsion by Arthur Kantrowitz in 1972 pioneering work in this area has been performed by a series of research groups worldwide $[1,2,3,4,5,6,7]$. For many years DLR investigated launch scenarios of small satellites [8]. However, the launch of nanosats (up to $10 \mathrm{~kg}$ wet mass) requires high power laser sources that still need significant development with respect to laser energy scaling and therefore are not available to the laser propulsion community.

In space, however, already existing lasers may be used as energy sources for the remote propulsion of small objects. Rezunkov et al [6] have already presented a laser propulsion engine exhibiting an optical assembly that provides a motion towards the laser source. A flexible combination of this concept with a conventional thruster device moving away from the laser source makes logistic applications possible. Power beaming for both acceleration and deceleration will allow for mail service in space or sample return missions to small planets or asteroids. 
Micro propulsion is a new research topic at DLR. In recent years the launch of numerous satellites for gravitational research had to be postponed due to the lack adequate thrust devices [9]. These missions require for extremely low thrust and high stability. Ablative micro propulsion employing onboard microchip lasers and momentum less beam control is a promising technology for low thrust devices.

Figure 1 illustrates the different laser propulsion regimes and the corresponding laser sources, yielding an almost constant impulse coupling coefficient $\mathrm{c}_{\mathrm{m}}$.

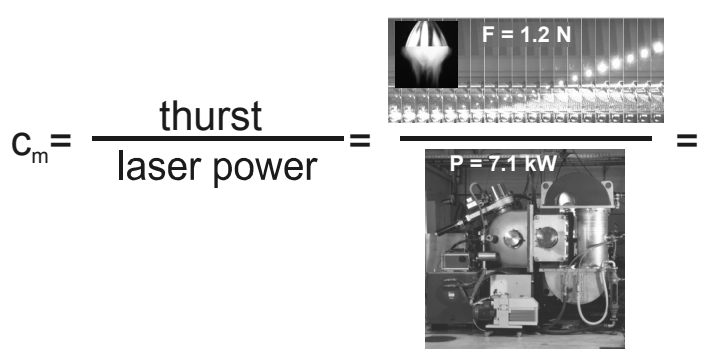

(a)

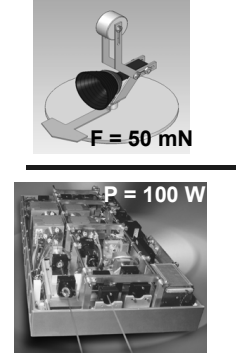

(b)

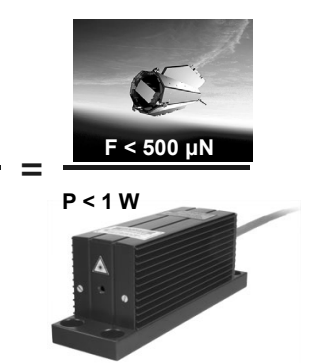

(c)

FIGURE 1. Laser propulsion research for different applications: (a) ground based launch of small satellites, (b) in space laser propulsion, and (c) ablative micro propulsion.

In the following the latest research activities at DLR in the different laser propulsion regimes will be briefly reported:

- A remotely controlled laser rocket with a thrust vector steering device integrated in a parabolic nozzle.

- Flight experiments with a parabolic thruster in an artificial 2D-zero gravity on an air cushion table.

- New investigations in the field of laser micro propulsion.

\section{REMOTELY CONTROLLED LASER ROCKET}

A simple configuration to control the ignition of a laser-supported detonation that can be used for a thrust vector steering device for a parabolic lightcraft has been developed at DLR. Free flights of laser-driven thrusters were mainly reported from the spin-stabilized Lightcraft Technology Demonstrator (LTD) of Leik Myrabo, among them the world-record flight with an altitude of 71 meters [2]. In the case of spinstabilization, however, the question arises how steering issues, e.g. orbit insertion, could be addressed. Nevertheless, the occurrence of unwanted lateral and angular velocity components due to limited back-driving forces raises the question of appropriate countermeasures. While the possibilities of varying the beam position at the lightcraft aperture are limited, an onboard solution has been found which offers additional steering potential for stability enhancement and orbit insertion [14].

During early lightcraft research at DLR, irregularities in detonations were found. These were ascribed to the high breakdown threshold of air $\left(1.5 \mathrm{GW} \cdot \mathrm{cm}^{-2}\right.$ at $10.6 \mu \mathrm{m}$ 

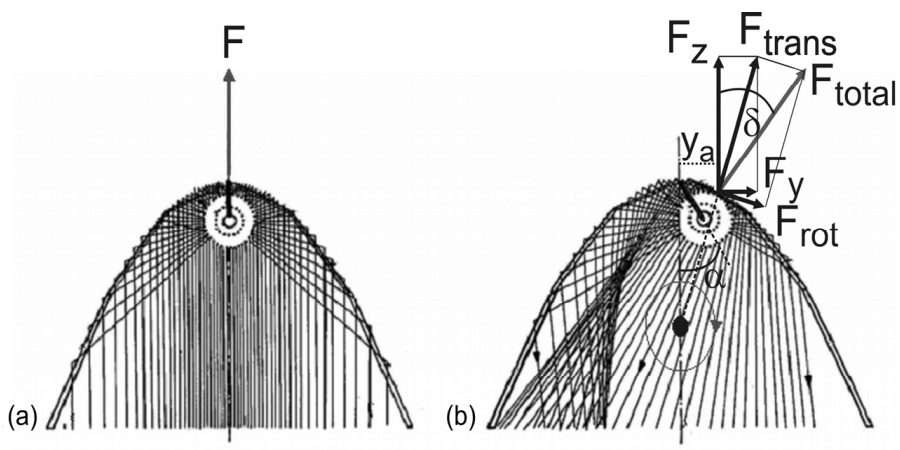

FIGURE 2. Propagation of detonation shock waves with coaxial (a) and tilted (b) ignition pin, as assumed in [11], together with the resulting thrust vector and its components giving axial and lateral impulse as well as angular momentum.

wavelength) compared to the low breakdown threshold $\left(1 \mathrm{MW} \cdot \mathrm{cm}^{-2}\right)$ in metal vapor close to a metallic surface [9]. Impurities on the reflector wall would also lower the ignition threshold, leading to a plasma detonation close to the mirror surface, but not in the focus. To provide for a lowered ignition threshold in the focus, a metallic ignition pin was introduced at the axis of symmetry (and patented [11]). This device allows for thrust vector steering by tilting the ignition pin and thus shifting the origin of the detonation. The effects of this pin and of a propellant rod in the same configuration were investigated. A theoretical analysis of the components of impulse coupling and the corresponding forces as well as a detailed description of the experimental setup can be found in [12].

\section{Rocket Construction}

A design model of a laser-driven rocket with a remotely controlled steering gear was constructed, as shown in Fig. 3 [13]. Inside the rocket we used a more robust reflector ( $1 \mathrm{~mm}$ thickness, $35.2 \mathrm{~g}$ mass) to prevent it from deformation. It was mounted in the lower part and connected to the steering unit in the center part. Two digital servos were placed on the steering platform perpendicular to each other enabling a tilt of the ignition rod in the nozzle by an angle of up to $10^{\circ}$ in the $x-y-$ plane. The remotely controlled servos were powered by LiPo batteries. The transmitter was operated from the operating desk. An additional payload can be placed at the upper platform of the rocket. The overall mass amounts to $154 \mathrm{~g}$ without payload.

\section{Results}

We investigated the data of intensity modeling to explore the shift of the detonation center. Initially we assumed that the center of detonation (COD) would be identical with the center of intensity (CI) and defined in analogy to the definition of the center of mass (CMS):

$$
\vec{r}_{C I}=\frac{\int I(\varphi, z) \vec{r} d V}{\int I(\varphi, z) d V} .
$$



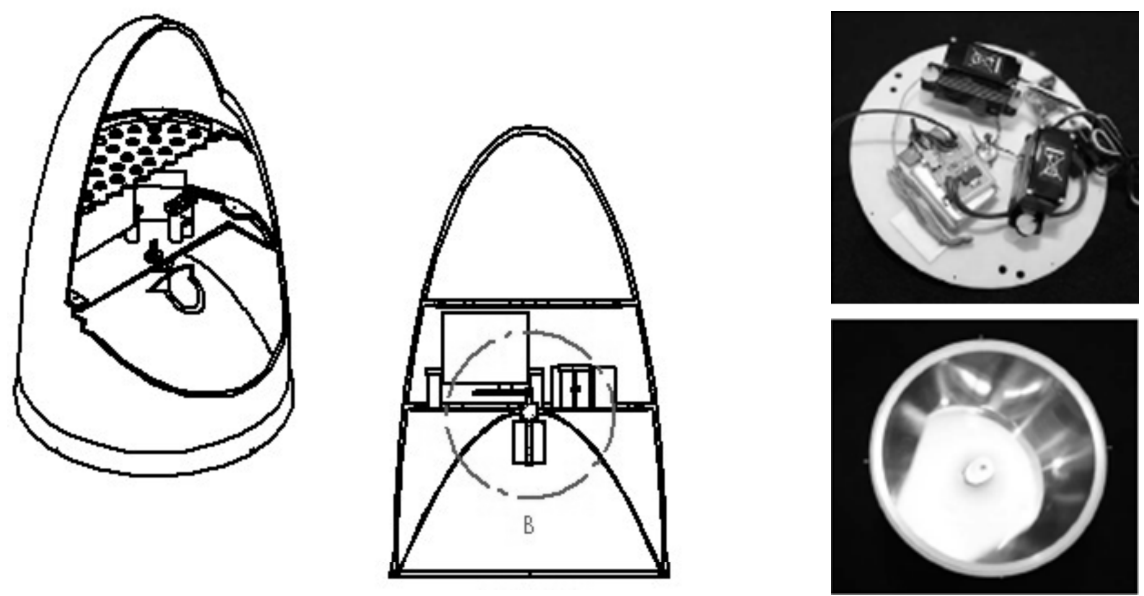

FIGURE 3. Conceptional sketch of the laser-driven rocket with a parabolic reflector/nozzle and a steering unit (lower platform) as well as components of the prototype.

Surprisingly the measurements of the thrust components in single pulse experiments and the reconstruction of the overall force vector [12] were in contrast to the preliminary expectations depicted in Fig. 2. The reconstruction of the overall force vector derived by the experimental data is summarized in Fig. 4. The negative lateral momentum and the negative lateral offset of the center of detonation suggest the introduction of a virtual center of detonation (COD) which is on the opposite side of the center of intensity $(\mathrm{CI})$.

Simple geometrical considerations, however, may shed some light on this process:

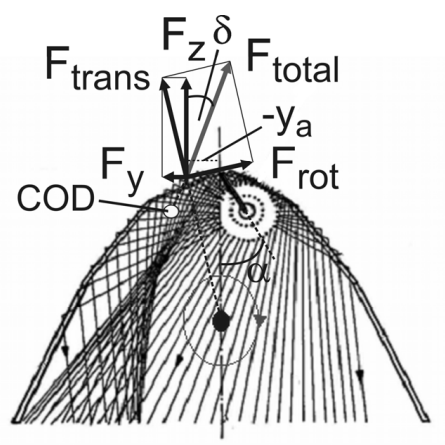

FIGURE 4. Reconstruction of the thrust vector, its components, and the virtual center of detonation (COD) according to the experimental results and conclusions from model data, in contrast to the preliminary assumptions [11], as displayed in Fig. 2.

On the opposite side of the rod, the detonation volume and the active surface of the nozzle are larger and may deliver a higher thrust. Results from modeling of the intensity distribution on the rod support these findings:

We assume a rod tilted to the right side of the lightcraft $(\alpha>0)$. The side of the rod that is closer to the lightcraft's symmetry axis (azimuthal angle $\varphi>0$ ) is mainly 
illuminated, since it is closer to the focus. According to the model setup in [14] this should induce a lateral momentum to the left $\left(c_{m, y}<0\right)$. Taking into account that the lateral differential force component should be diminished by $\cos \alpha$ due to the tilt of the propellant rod and jet, we find as a coarse approximation:

$$
F_{y} \propto \cos \alpha \int I(\varphi) \sin \varphi d \varphi
$$

The derived values for the lateral force component are depicted in Fig. 5. They are in a fairly good accordance with the experimental results. Furthermore, these findings appear to be similar to the results of [14], where at the side of the ignition pin with the most intense fluence a lateral force component occurred. However, this is a rough simplification, since in [14] we presume the differential force components to be independent of $\varphi$ with respect to their point of action and tilt angle relative to the lightcraft's symmetry axis. For the case of a tilted rod, a more elaborate model is needed.

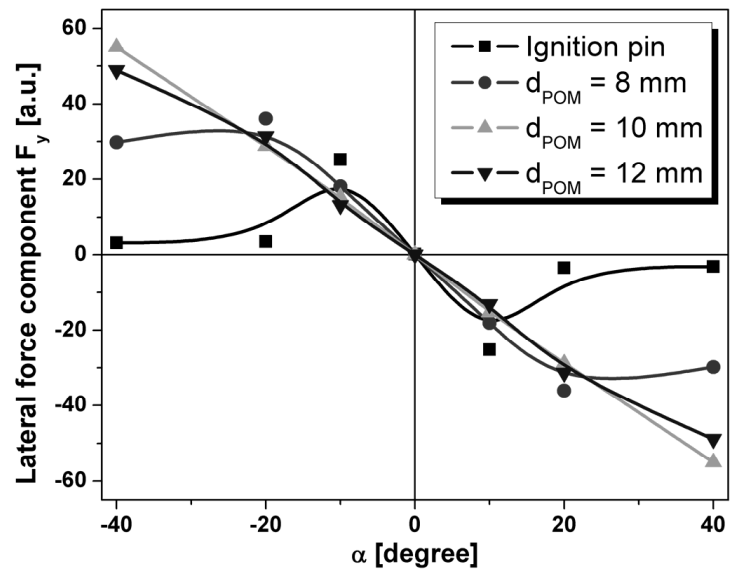

FIGURE 5. Theoretical data for the lateral force component from intensity modeling.

First test flights with an average laser power of $3.2 \pm 0.3 \mathrm{~kW}$ and $3.1 \pm 0.3 \mathrm{~kW}$, demonstrated the practical feasibility of thrust vector steering by changing the ignition configuration, cf. Fig. 6 . The performance of the steering unit was not significantly affected by the strong accelerations during the laser pulse.

The laser power was sufficient to lift the thruster. Nevertheless, in the temporal course of the laser burst, the rocket descends again. Since the propagation path of the laser beam is aligned to vertical, in a first approach this effect has been ascribed to the reduced rate of energy entering the lightcraft with increasing inclination angle and lateral offset of the inlet. The energy losses have been modeled by a ray-tracing algorithm, only yielding a $10 \%$ reduction of $c_{m \text {,eff }}[14]$.Thus, the decrease during the flight is supposed to be due to significant changes of the intensity distribution on the 
propellant rod under inclined illumination. These topics will be the subject of further research.
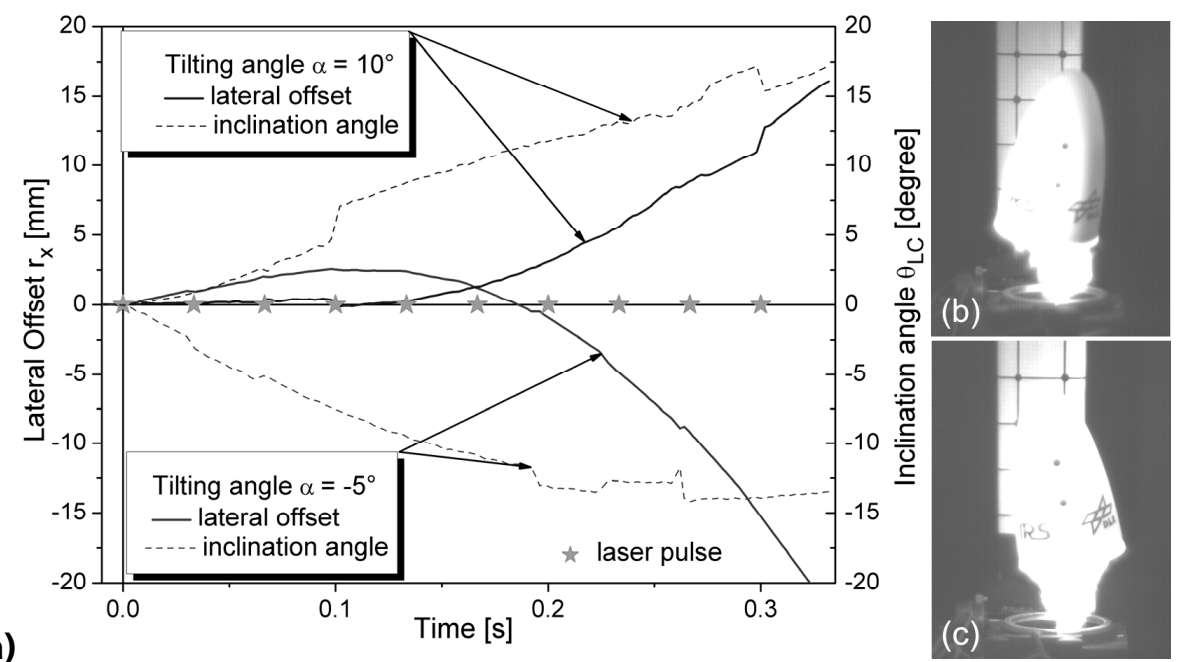

FIGURE 6. (a) Lateral offset (solid) of the center of mass and inclination angle (dashed) of a laserdriven rocket (mass: $154 \mathrm{~g}$ ) with remotely accessible steering gear in a multiple pulse flight for different tilting angles of the propellant rod. On the right frames from high speed recording of laser pulse \# 6 with positive (b) and negative (c) inclination are depicted.

\section{LASER PROPULSION IN 2D-ZERO GRAVITY}

The exploration of in-space laser propulsion in artificial weightlessness is a first step to space-borne applications of laser propulsion. First ideas employing the drop tower facility in Bremen have been reported in [15] but had to be postponed due to limited budgets. An alternative approach is the investigation of energy scaling in $2 \mathrm{D}$ artificial weightlessness on an air cushion table by scaling down the dimensions of a

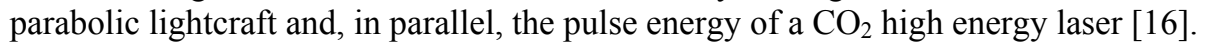

\section{Theoretical Basis}

For several thruster geometries, laser heating of the plasma in a shockwave front during the laser pulse has been approximated according to the hydrodynamic model of a point explosion with counter-pressure [17]. It was adapted by V. P. Ageev for laser propulsion with various thruster geometries [18]. The dynamic radius $R_{0}$ of the explosion

$$
R_{0}=\sqrt[3]{\frac{E}{p_{0}}}
$$


and the geometric parameters $R$ (focal length) and $D$ (aperture diameter) of the impulse receiver; i.e., the reflector and nozzle, determine the impulse coupling coefficient $c_{m}$ of the laser pulse:

$$
c_{m}=\frac{4 \pi}{c_{0}} \frac{R_{1}^{2}-R^{2}}{R_{0}^{2}} J^{(1)}\left(\frac{R_{1}}{R_{0}}\right),
$$

with

$$
R_{1}=R \cdot\left[1+\left(\frac{4 R}{D}\right)^{-2}\right],
$$

where $p_{0}$ denotes the ambient pressure, $c_{0}$ the speed of sound and $J^{(1)}(x)$ the dimensionless impulse, as tabulated in [17]. It is important to note that $E$ is the energy deposited in a point explosion in general. In our case, $E$ is the laser pulse energy, regardless of wavelength and pulse length. This simple approximation does not include effects like the temporal behavior of the laser plasma interaction.

\section{Experiment}

Parabolic nozzle geometries that have been optimized for low pulse energies have already been presented in [15]. Their features are listed in Table 1.

\begin{tabular}{|c|c|c|c|c|c|}
\hline Type & $\begin{array}{c}\text { Diameter D } \\
\mathbf{m m}\end{array}$ & $\begin{array}{c}\text { Focal length } R \\
\mathrm{~mm}\end{array}$ & Geometric Parameter R/D & $\begin{array}{c}\text { Height } \\
\text { mm }\end{array}$ & $\begin{array}{c}\text { Mass } \\
\text { g }\end{array}$ \\
\hline 1 & 30 & 2.56 & 0.085 & 21.9 & 3.16 \\
\hline 2 & 20 & 1.54 & 0.077 & 16.2 & 1.25 \\
\hline 3 & 20 & 2.85 & 0.143 & 8.8 & 1.10 \\
\hline 4 & 10 & 1.22 & 0.122 & 5.1 & 0.22 \\
\hline \multicolumn{6}{|c|}{ for comparison: Lightcraft used for ground-based launch experiments in [21] } \\
\hline & 100 & 10 & 0.1 & 62.5 & 19 \\
\hline
\end{tabular}

TABLE 1. Lightcraft design parameters for experiments in weightlessness

(a)

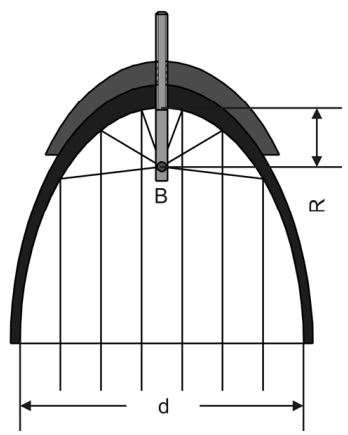

(b)

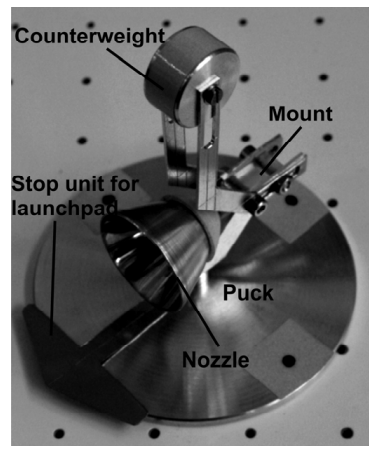

FIGURE 7. Lightcraft nozzle, (a) scheme with cap and propellant rod and (b) mounted on a puck with tracking markers. A counter-weight to adjust the center of mass is placed on top. 
Each nozzle was fixed to an adjustable mounting assembly on a puck for hovering on the air cushion table. Details on this setup, the corresponding measuring technique, and the data analysis are described in $[14,16,19,20]$

\section{Results and Discussion}

Air breakdown in single pulse experiments as well as laser ablation investigations have been carried out. The results for coupling coefficients in a single pulse experiment on the air cushion table are depicted in Fig. 7. Inside the nozzle, neither an ignition pin nor a propellant were used. The portion of the laser pulse energy entering the lightcraft's aperture ranged from 3 to $21 \mathrm{~J}$ (type 1), 1 to $10 \mathrm{~J}$ (types 2 and 3), and 1 to $2.3 \mathrm{~J}$ (type 4 ), resp. With each parameter setup, five experiments have been performed.

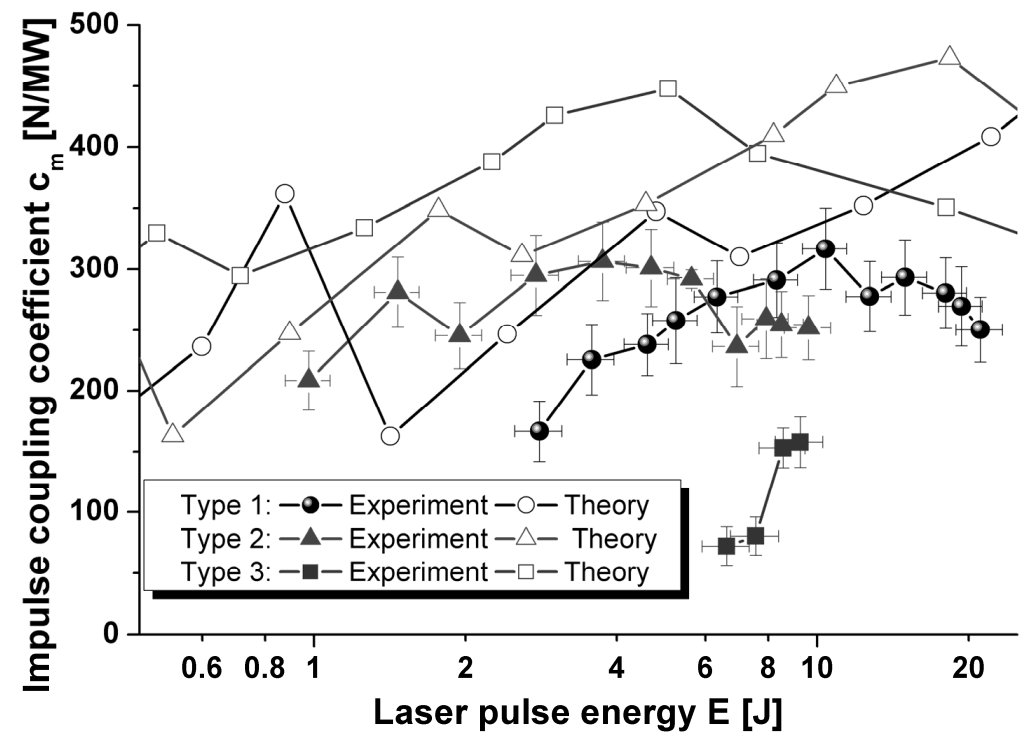

FIGURE 8. Data from measurements at the air cushion table: Coupling coefficients of different nozzles, cf. Table 1, mounted on a puck vs. laser pulse energy and theoretical values (open symbols), derived by the theory of point explosion with counter-pressure. Data from type 4 were not measurable, since no air breakdown was achieved.

For low pulse energies, the data of type 1 and 2 are in quite good agreement with theoretical values. However, at higher pulses energies $(E>11 \mathrm{~J}$, type 1 , and $E>5 \mathrm{~J}$, type 2), the experimental findings were much lower than the theoretical predictions. This effect can be attributed to energy loss due to internal vibrations of the flyer. Though only a sparse database of theoretical values had been computed, even the predicted local maxima in the course of $c_{m}$ (at $4.8 \mathrm{~J}$ - type 1 , at $1.8 \mathrm{~J}$ - type 2) seem to be verified by the experiment, however, within the range of the corresponding error bars and with a lateral shift of the experimental data graph (Fig. 8). 


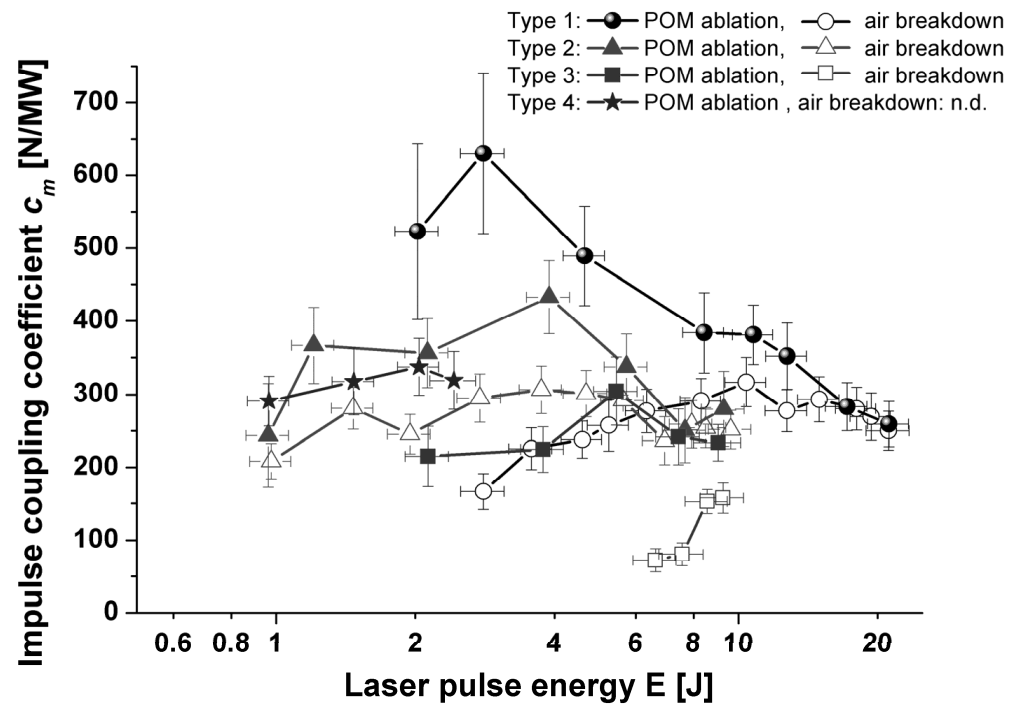

FIGURE 9. Data from measurements at the air cushion table: Coupling coefficients of different parabolic mirrors, cf. Table 1, with a propellant rod of Delrin ${ }^{\circledR}$ in the focus. Open symbols indicate data from comparative measurements without propellant.

The single pulse experiments have been repeated with a rod of POM mounted on the symmetry axis of the nozzle. POM ablation from a circular focal zone enhanced the momentum by a factor of 2 to $3 \mathrm{cf}$. Fig. 9, as already found for large parabolic nozzles in this ablation geometry [8]. At higher pulse energies, however, this effect seems to be limited, cf. the data for POM vs. air breakdown of nozzle 1 ( $E>10 \mathrm{~J})$, and nozzle $2(E>5 \mathrm{~J})$. Again, this effect may be ascribed to energy losses in structural vibrations of the puck at high pulse energies.

Finally we compared our results with data from experiments using a vertical launch setup. Due to gravity, the limit of detection was higher than at the air cushion table, even with the larger mass of the puck. Since the small, lightweight nozzles exhibited only poor beam-riding abilities, an extensive comparison of multiple-pulsed flights between the two different setups was not possible.

A more detailed discussion of all the different experiments in artificial 2Dweightlessness is given in [19].

\section{ABLATIVE MICRO PROPULSION}

Alternative thruster technologies are required for missions involving micro spacecrafts or station-keeping on very low, but precise thrust levels. Especially scientific missions for mapping the Earth's magnetic or gravity fields and for the detection of gravitational waves, e.g., CHAMP, GRACE, GOCE, MICROSCOPE or 
LISA [22], require new thruster concepts. These concepts should also provide for long term reliability and high efficiency. Lasers have proven their long term reliability in many applications, e.g., in industry or medicine. Depending on the laser power, pulsed laser ablation of metals or polymers reveals a broad range of impulse bits and corresponding average thrust, as defined by the momentum coupling coefficient $[23,24]$.

As a first step towards laser micro propulsion, experiments using a highly repetitive $\mathrm{Nd}: \mathrm{YVO}_{4}$ laser are in preparation. The goal during this first phase is to identify and characterize suitable materials for laser ablation. For this purpose, a high accuracy thrust stand is under development.

The resulting data will also be used for the selection of a laser source to be optimized for space applications by tailoring laser pulse length and shape with respect to repetition rate, fluence on target and a suitable wavelength for ablation.

The implementation of the propulsion concept includes research on momentum-less beam control and tests on technological reliability (Fig. 10). Additionally, SEM and time resolved imaging of the plasma plume will be applied for analysis of reproducibility and directionality of the ablation jet.

In addition to experiments on long term stability of the microthruster, an experiment under realistic conditions (microgravity, vacuum) is planned to be carried out at the ZARM drop tower at the University of Bremen in order to demonstrate the feasibility and maturity of this technique.

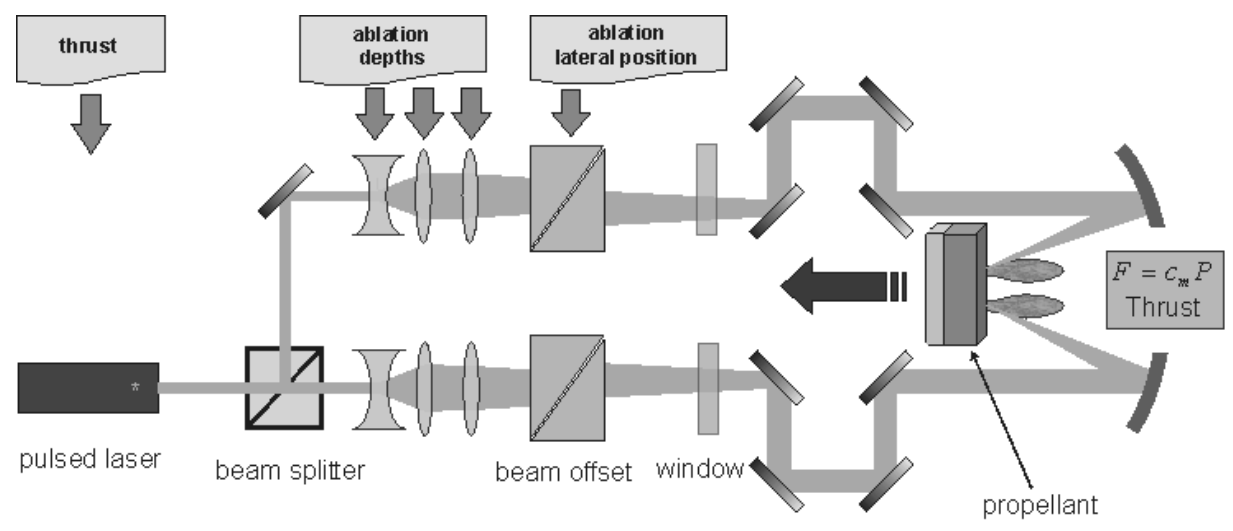

FIGURE 10. Concept of a laser ablation micro propulsion device with momentum-less beam control.

\section{CONCLUSION}

Despite many worldwide efforts in research the laser propulsion community has not been able to establish a satellite launch project. This has to be attributed to the lack of suitable laser sources. There are two alternatives to evade this gap. Researchers may stick to small scale experiments studying limited aspects of future propulsion projects or investigate laser propulsion in a zero gravity environment. In the latter case lower thrust levels are required and therefore sufficient laser sources are available. 
DLR still has research activities in both fields. For the satellite launch scenario we investigated the feasibility of thrust vector steering of a laser lightcraft by variation of its ignition configuration. Direction, magnitude, and point of action of the thrust vector are dependent on various parameters, a situation which demands a more detailed analysis. A tilted configuration of a propellant rod inside the thruster induces lateral and angular momentum. A detailed analysis led to the introduction of a virtual center of the detonation. The remote control of the steering device allows for stabilization of the flight and is a prerequisite for a closed loop flight control based on optical tracking.

As a first step to the zero gravity regime the high speed analysis of pulsed horizontal "flights" of a parabolic thruster on an air cushion table allowed for measurement of the impulse coupling coefficient with respect to three degrees of freedom. The results for translational momentum coupling in a single pulse experiment correspond with theoretical values as well as with averaged data from multiple pulsed flights. The lower velocity increment per pulse compared to vertical flights allows for a detailed analysis of several subsequent pulses on a stretched timescale.

The air cushion table experiments proved to be a useful substitute for in-space propulsion. However, they were performed in ambient air and motion in weightlessness was only achieved for three degrees of freedom instead of six. As already shown with the free flights in 2000 by Leik Myrabo [2], experiments for demonstration purposes have the greatest impact when the experimental scenario is close to reality.

Another approach to the investigation of laser propulsion in space is laser ablative micro propulsion. Since there is a strong demand for thrusters on the $\mu \mathrm{N}$ to $\mathrm{mN}$ level, the development of spaceproof laser sources and laser-driven micro thrusters will become an increasing activity at DLR. A micro propulsion demonstration experiment in microgravity, as already proposed in [15], is under preparation.

\section{ACKNOWLEDGMENTS}

The authors want to thank Stephanie Karg for fruitful discussions.

\section{REFERENCES}

1. A. Kantrowitz, "Propulsion to orbit by ground-based laser", Astronautics \& Aeronautics, 1972, 10, $74-76$.

2. L. N. Myrabo, "World Record Flights of beam-Riding Rocket Lightcraft: Demonstration of "Disruptive" Propulsion Technology", in 37th AIAA/ASME/SAE/ASEE Joint Propulsion Conference, 8-11 July 2001, Salt Lake City, 2001-3798.

3. C. W. Larson, F. B. Mead, Jr., and S. D. Knecht "Laser Propulsion and the Constant Momentum Mission" in Beamed Energy Propulsion, edited by K. Komurasaki, AIP Conference Proceedings 702, American Institute of Physics, Melville, NY, 2004, pp. 216-227.

4. C. R. Phipps et al. "ORION: Clearing near-Earth Space Debris using a 20 kW, 530-nm, EarthBased, Repetitively Pulsed Laser" in Laser and Particle Beams, Vol. 14, No 1, 1996, pp. 1-44.

5. A. V. Pakhomov at al "Ablative Laser Propulsion: An Update, Part I, in Beamed Energy Propulsion, edited by K. Komurasaki, AIP Conference Proceedings 702, American Institute of Physics, Melville, NY, 2004, pp. 166-177. 
6. Yu.A. Rezunkov, A.L. Safronov, A.A. Ageichik, M.S. Egorov, V.V. Stepanov, V.S.Rachuk, V.Yu. Guerman, A.V. Ivano, S.G. Rebrov, and A.N. Golikov, "Performance Characteristics of Laser Propulsion Engine Operating both in CW and in Repetitively-Pulsed Modes", in Beamed Energy Propulsion, edited by K. Komurasaki, T. Yabe, S. Uchida, and A. Sasoh, AIP Conference Proceedings 830, American Institute of Physics, Melville, NY, 2006, pp. 3 - 13.

7. A. Sasoh, N. Urabe, S. Kim, "Impulse enhancement by in-tube operation in laser propulsion", High-Power Laser Ablation IV, Proc. of SPIE, Vol. 4760, 2002, pp. 879-886.

8. W. L. Bohn and W. O. Schall, "Laser Propulsion Activities in Germany" in Beamed Energy Propulsion, edited by A. V. Pakhomov, AIP Conference Proceedings 664, American Institute of Physics, Melville, NY, 2002, pp. 79-91

9. P. Touboul, "The MICROSCOPE mission present status", From quantum to cosmos $I V$, Bremen, (21.-24. September 2009)

10. H.M. Musal, "Pulsed Laser Initiation of Surface Plasma on Metal Mirrors", in Laser Induced Damage In Optical Materials: 1980, edited by H.E. Bennett et al., NBS Special Publication 620, NBS, Boulder, Colorado, 1981, p. 227, cited in: [11].

11. W.O. Schall, E. Zeyfang, W. Riede and W. Mayerhofer, "Propulsion device and method of generating shock waves", Patent DE 10017343 C2 (7 April 2000) (in German) and U.S. Patent No. 2002/0047673 A1 (6 April 2001).

12. S. Scharring, D. Hoffmann, H.-A. Eckel and H.-P. Röser, Acta Astronautica 65, 1599-1615 (2009).

13. D. Hoffmann, "Development and validation of a design model with remotely controllable steering gear for flight experiments on pulsed laser thermal propulsion", Diploma thesis, IRS-07-S39, University of Stuttgart, 2008.

14. S. Scharring, H.-A. Eckel, and H.-P Röser, "High speed Analysis of Free Flights with a Parabolic Thruster", in Beamed Energy Propulsion, edited by C. R. Phipps, K. Komurasaki, J. E. Sinko, AIP Conference Proceedings, American Institute of Physics, Melville, NY, 2009, to be published.

15. S. Scharring, H.-A. Eckel, J. Trommer, H.-P Röser, and Ch. Eigenbrod, "Spaceborne Lightcraft Applications - an Experimental Approach", in Beamed Energy Propulsion, edited by A. V. Pakhomov, AIP Conference Proceedings 997, American Institute of Physics, Melville, NY, 2008, pp. 295-303.

16. E. Wollenhaupt, "Experimental studies on the scaling of laser-thermal propulsion", Diploma thesis, IRS-09-S12, University of Stuttgart, 2009 (in German).

17. D.E. Ochozimskij, I.L. Kondrasheva, S.Z. Vlasova, R.K. Kasakova, "Calculation of a Point Explosion with Respect to Counterpressure" in Akademia Nauk SSSR 1957, pp. 1-65 (in Russian).

18. V.P. Ageev, A.I. Barchukov, F.V. Bunkin, V.I. Konov, A.M. Prokhorov, A.S. Silenok, N.I. Chapliev, Soviet Journal of Quantum Electronics 7(12), 1430-1437 (1977).

19. S. Scharring, H.-A. Eckel, H.-P. Röser, "Flight Experiments on Energy Scaling for In-Space Laser Propulsion" in Beamed Energy Propulsion, edited by C. R. Phipps, K. Komurasaki, J. E. Sinko, AIP Conference Proceedings, American Institute of Physics, Melville, NY, 2009, to be published.

20. J. Sinko, S. Scharring, H.-A. Eckel, H.-P. Röser, and A. Sasoh, "Measurement Issues in Pulsed Laser Propulsion" in Beamed Energy Propulsion, edited by C. R. Phipps, K. Komurasaki, J. E. Sinko, AIP Conference Proceedings, American Institute of Physics, Melville, NY, 2009, to be published.

21. S. Scharring, H.-A. Eckel, and H.-P Röser, "Flight Analysis of a Parabolic Lightcraft - Groundbased Launch", in Beamed Energy Propulsion, edited by A. V. Pakhomov, AIP Conference Proceedings 997, American Institute of Physics, Melville, NY, 2008, pp. 304-315.

22. M. Rodrigues, B. Foulon, F. Liorzou, and P. Touboul, "Flight experience on CHAMP and GRACE with ultra-sensitive accelerometers and return for LISA", Class. Quantum Grav. 20, 291-300 (2003)

23. Phipps, C.R., Luke, J.R., and Helgeson, W., 3 ks specific impulse with a ns-pulse laser microthruster, paper IEPC 319, 29th International Electric Propulsion Conference, Princeton (2005)

24. Phipps, C.R., Luke, J.R., and Helgeson, W., Giant momentum coupling coefficients from nanoscale laser-initiated exothermic compounds, paper AIAA 2005-3607, 41st AIAA/ASME/SAE/ASEE Joint Propulsion Conference, Tucson (2005) 